\title{
RELATIVISTIC PARTICLE TRAJECTORIES FROM WORLDLINE PATH INTEGRAL QUANTIZATION
}

\author{
Philip R. Johnson*† \\ University of Maryland, College Park, MD 20742, USA
}

\section{Abstract}

Using the worldline path integral quantization framework in quantum field theory we construct a generating functional for a relativistic particle's quantum-average trajectory. The approach systematically generalizes to incorporate quantum corrections (to the average) and quantum fluctuations (around the average).

\section{INTRODUCTION}

Classical mechanics computes trajectories in phase space: unique solutions to differential equations yielding precise positions and momenta. Quantum mechanics traditionally makes little use of the concept of trajectory since a system's state never corresponds to a definite worldline through spacetime. In nonrelativistic quantum mechanics there is at least the formal connection between classical and quantum descriptions of motion involving the replacement of classical positions and momenta with operators obeying the operator version of Hamilton's equations of motion.

But in relativistic quantum field theory the notion of trajectory becomes even more removed since there is no good position operator $\hat{x}$. The standard procedure demotes the coordinate $x$ from a dynamical variable altogether by introducing the operator-valued fields $(\hat{\psi}(\mathbf{x}, t), \hat{\pi}(\mathbf{x}, t))$ whose equations of motion are then generated by a Hamiltonian $\hat{H}=\hat{H}(\hat{\psi}, \hat{\pi})$. In the field theoretic approach a relativistic particle's average position is computed using the fact that $\left\langle i n\left|\hat{\jmath}^{0}(\mathbf{x}, t)\right| i n\right\rangle$ is proportional to the probability density for the state $|i n\rangle$, where $\hat{\jmath}^{0}(\mathbf{x}, t)$ is the time-component of a conserved-current operator obeying the Heisenberg equations of motion: $i \partial_{t} \hat{\jmath}^{\mu}(\mathbf{x}, t)=\left[\hat{\jmath}^{\mu}(\mathbf{x}, t), \hat{H}\right]$. We describe the computation (and simulation) of the four-current expectation value (though in the Schrödinger rather than Heisenberg pictures) in [1].

However there is an alternative to the standard method of quantizing relativistic systems via quantum fields going back at least as far as Feynman and Schwinger. This alternative approach has been studied in detail as an example of a reparametrization invariant theory both in the context of General Relativity and String theory. Going by the name of 'worldline quantization' it has also found practical applications to certain problems in QED and QCD [2].

This paper outlines how path integral quantization of a particle's worldline leads to a very direct framework for computing equations of motion for the expectations values corresponding to a particle's average trajectory (i.e.

*philipj@physics.umd.edu

† Work supported in part by DOE Grant DEFG0296ER40949. the semiclassical equations of motion), and the fluctuations around that trajectory. Under certain conditions, incoherent quantum fluctuations well-approximated by classical statistics are important (e.g. synchrotron radiation induced emittance growth). In such a regime, where the semiclassical approximation is inadequate but coherent quantum effects aren't manifestly important, the dynamics of particle correlation functions are to a good approximation described by stochastic trajectories. Stochastic equations of motion are certainly more work to solve than purely (deterministic) classical equations, but they are still far less work (computationally) than solving for the full quantum behavior.

\section{WORLDLINE QUANTIZATION}

The basic idea starts with this: rather than demote the particle coordinate $x$ to a mere parameter, promote the particle time coordinate $t$ to an operator whose conjugate is the energy $p_{0}$ (we set $\hbar=c=1$ ). The canonical commutation relations become $\left[\hat{x}_{\mu}, \hat{p}_{v}\right]=i g_{\mu \nu}$, where $g_{\mu \nu}=\operatorname{diag}(1,-1,-$ $1,-1)$ is the Lorentzian metric. Hence, we construct a model of quantized worldline operators $\hat{x}^{\mu}(\tau)$ and $\hat{p}_{\nu}(\tau)$. A new parameter $\tau$ is introduced, but physical observables are invariant under reparametrizations $\tau \rightarrow \tau^{\prime}(\tau)=\tau+\varepsilon(\tau)$ making the quantized worldline a gauge theory.

Ordinarily, one chooses the quantity $S[x]=m \int d s$ proportional to the propertime along the worldline as a manifestly Lorentz invariant action for a (spinless) relativistic particle, but the implicit square root complicates matters in the quantum theory. An alternative (in fact a more general) choice of the action is

$$
S[x, N]=\int d \tau\left(\frac{\dot{x}^{2}}{2 N}-\frac{N m^{2}}{2}\right),
$$

where $N(\tau)$ is a Lagrange multiplier. One may immediately verify both that $p_{\mu}=\dot{x}_{\mu} / N$ and that $\delta S / \delta N=$ $\delta S / \delta x^{\mu}=0$ give the classical equations of motion including the mass-shell constraint $p^{2}=m^{2}$. The Hamiltonian is $H=N\left(p^{2}-m^{2}\right) / 2$. In the quantized theory $N$ also become an operator. For simplicity we neglect the field dynamics in this paper.

Now the quantized worldline theory can be treated as essentially an ordinary quantum mechanical theory (with $\tau$ the "time" parameter), though there is still the complicating presence of the mass-shell operator constraint $\hat{p}^{2}-m^{2}=$ 0 , and the reparametrization (gauge) symmetry. Basically, the constraint requires that any physical states in Hilbert space satisfy

$$
\left(\hat{p}^{2}-m^{2}\right)|p h y s\rangle=0
$$


On-shell momentum states $|\mathbf{p}, \pm\rangle \equiv\left|p_{0}= \pm\left(\mathbf{p}^{2}+m^{2}\right), \mathbf{p}\right\rangle$ easily satisfy this condition since $\hat{p}_{\mu}|p\rangle=p_{\mu}|p\rangle$ and $p_{0}^{2}=\mathbf{p}^{2}+m^{2}$. Note that the constraint allows positive and negative energy states. On the other hand, no worldline configuration-space states $|x\rangle=\left|x^{0}, \mathbf{x}\right\rangle$ are physical since

$$
\left\langle x^{\prime}\left|\left(\hat{p}^{2}-m^{2}\right)\right| x\right\rangle=-\left(\partial^{\mu} \partial_{\mu}+m^{2}\right) \delta^{(4)}\left(x-x^{\prime}\right) \neq 0
$$

clearly implies $\left(\hat{p}^{2}+m^{2}\right)|x\rangle \neq 0$ (we've used $\hat{p}_{\mu} \rightarrow$ $-i \partial_{\mu}$ ). Therefore, even though we have regained a relativistic position operator $\hat{x}^{\mu}$ by this approach, its eigenstates are not physical and hence we still don't have localized single particle states of the nonrelativistic kind. But, while the states $|x\rangle$ are not physical, they do span a Hilbert space $\mathcal{H}$ that has the physical Hilbert space $\mathcal{H}_{\text {phys }}$, spanned by $| \pm, \mathbf{p}\rangle$, as a subspace. We are free to make use of $|x\rangle$ so long as we ensure that the evolving physical state of the system always stays within $\mathcal{H}_{\text {phys }}$. The states $|x, N\rangle=|x\rangle \otimes|N\rangle$ give

$$
I=\int d x d N|x, N\rangle\langle x, N|
$$

as a representation of the identity in $\mathcal{H}$.

Next we discuss how these facts allow for the standard 'quantum mechanical' path integral quantization of the relativistic particle leading to a systematic framework for computing expectation values that are the origin of trajectories in classical physics.

\section{PATH INTEGRAL QUANTIZATION}

A sum over histories representation for the propagator $U\left(x_{2}, x_{1}\right) \equiv\left\langle x_{2}, N_{2} \mid x_{1}, N_{1}\right\rangle$ may be found when the Green's function obeys the composition rule

$$
\begin{aligned}
U\left(x_{2}, N_{2} ; x_{1}, N_{1}\right)= & \int d x_{3} d N_{3} U\left(x_{2}, N_{2} ; x_{3}, N_{1}\right) \\
& \times U\left(x_{3}, N_{3} ; x_{1}, N_{1}\right) .
\end{aligned}
$$

This requirement is essentially equivalent to there existing a representation of the identity of the form Eq. 1. Consequently, the relativistic worldline may be quantized by the rule

$$
U\left(x_{2}, x_{1}\right)=\int_{x_{1}, N_{1}}^{x_{2}, N_{2}} D x D N e^{i S[x, N]} .
$$

We define

$$
D x D N=\Pi_{\mu} \Pi_{i=1}^{N} \int d x^{\mu}\left(\tau_{i}\right) d N\left(\tau_{i}\right),
$$

where the worldline parameter $\tau$ is discretized as $\left(\tau_{1}, \tau_{2}, \ldots, \tau_{N}\right)$ and $N \rightarrow \infty$ in the continuum limit.

However, because of the reparametrization invariance of the action $S[x, N]$, Eq. 2 sums over an infinite number of gauge-equivalent trajectories. The cure is to pick a gauge so that one only sums over physically inequivalent trajectories. A non-covariant gauge choice sets $t=\tau$ (this gauge is implicitly used in [1]). A covariant choice is $N=$ constant implying that $d \tau$ is proportional to the propertime $d s$. However, this does not fully fix the $N(\tau)$ path integral because one must still sum over the possible values of the constant $N$. After gauge-fixing the propagator can be put in the form

$$
U\left(x_{2} ; x_{1}\right)=\int_{0}^{\infty} d N \int_{x_{1}}^{x_{2}} D x e^{\frac{i}{2} \int_{0}^{N}\left(\dot{x}^{2}-m^{2}\right) d \tau} .
$$

This may be interpreted as first a sum over every possible worldline that goes from $x_{1}$ to $x_{2}$ in propertime $N$, and then a sum over all (positive) propertimes. The choice of limits on the $N$ integral is related to the boundary conditions and picks out the Feynman propagator, which one may directly verify. The worldlines in the sum include those that go faster than light and even backward-in-time.

\section{WORLDLINE GENERATING FUNCTIONAL AND TRAJECTORIES}

The path integral formulation, while intuitively appealing, has still not giving us equations of motion for a trajectory. We have so far been following the standard development such as may be found in $[2,3]$. Our next step adapts this framework to the computation of trajectories and their fluctuations. We first add a source term to the action giving $S[\rho]=S[x, N]+\int d \tau \rho_{\mu}(\tau) \dot{x}^{\mu}(\tau)$. With this modification, Eq. 3 becomes a generating functional $Z[\rho]$. Using $Z[\rho]$ we find the correlation functions

$$
\begin{aligned}
\left.\frac{\delta Z[\rho]}{\delta \rho\left(\tau_{1}\right)}\right|_{\rho=0} & =\int d N \int D x \dot{x}\left(\tau_{1}\right) e^{\frac{i}{2} \int_{0}^{N}\left(\dot{x}^{2}-m^{2}\right) d \tau} \\
& =i\left\langle x_{2}\left|d \hat{x}^{\mu} / d \tau_{1}\right| x_{1}\right\rangle \\
\left.\frac{\delta^{2} Z[\rho]}{\delta \rho_{1} \delta \rho_{2}}\right|_{\rho=0} & =\int d N \int D x \dot{x}_{1} \dot{x}_{2} e^{\frac{i}{2} \int_{0}^{N}\left(\dot{x}^{2}-m^{2}\right) d \tau} \\
& =i^{2}\left\langle x_{2}\left|\left(d \hat{x}^{\mu} / d \tau_{1}\right)\left(d \hat{x}^{\mu} / d \tau_{2}\right)\right| x_{1}\right\rangle
\end{aligned}
$$

and so on for the higher-order correlation functions. However, Eq. 4 does not give the true average particle trajectory because it requires specifying both an initial and final particle state, $\left|x_{1}\right\rangle$ and $\left|x_{2}\right\rangle$, respectively. Moreover, it may be shown that the equations of motion from Eq. 4 are generally neither real nor causal .

The solution is to define a so-called in-in generating functional

$$
\begin{aligned}
Z\left[\rho, \rho^{\prime}\right] \equiv & \int d x_{1}^{\prime} d x_{1} d x_{2} \psi_{i n}^{*}\left(x_{1}^{\prime}\right) U\left(x_{1}^{\prime}, x_{2}\right)_{\rho^{\prime}} \\
& \times U\left(x_{2}, x_{1}\right)_{\rho} \psi_{i n}\left(x_{1}\right) \\
= & \int d x_{1}^{\prime} d x_{1} d x_{2} \int d N d N^{\prime} \int_{x_{1}^{\prime}, x_{1}}^{x_{2}} D x^{\prime} D x \\
& e^{i(S-S)} e^{i \int\left(\rho \dot{x}-\rho^{\prime} \dot{x}^{\prime}\right) d \tau} \psi_{i n}^{*}\left(x_{1}^{\prime}\right) \psi_{i n}\left(x_{1}\right) .
\end{aligned}
$$


Then it follows that

$$
\left.\frac{\delta Z\left[\rho, \rho^{\prime}\right]}{\delta \rho_{1}}\right|_{\rho, \rho^{\prime}=0}=i\left\langle\psi_{i n}\left|d \hat{x}^{\mu} / d \tau_{1}\right| \psi_{i n}\right\rangle,
$$

where $\left|\psi_{i n}\right\rangle$ is the initial state. The higher-order correlation functions also only refer to the initial state. Therefore, $Z\left[\rho, \rho^{\prime}\right]$ is the generating functional for true expectation values and the resulting equations of motion for $\left\langle d \hat{x}^{\mu} / d \tau_{1}\right\rangle$ may be shown to be both real and causal [4]. We may use the generating functional $W\left[\rho, \rho^{\prime}\right]=-i \ln Z\left[\rho, \rho^{\prime}\right]$ to define normalized expectation values. In terms of Feynman diagrams, defining $W$ is equivalent to factoring out vacuum bubbles leaving only connected diagrams.

Returning to the path integral Eq. 3, it is a familiar result that the solution is dominated by the extremal solution to the action because the classical worldline (and the worldlines near it) give coherently reinforcing phases in the integrand. The semiclassical (i.e. loop) expansion takes advantage of this fact by defining deviation variables $\tilde{x}=x-x_{0}$ and $\tilde{N}=N-N_{0}$, where $x_{0}, N_{0}$ are the extremal solutions found from $\delta S[\rho] / \delta x_{0}=\delta S[\rho] / \delta N_{0}=0$. They are functions of the sources $\rho$ and $\rho^{\prime}$. We similarly define deviation variables for $x^{\prime}, N^{\prime}$. Somewhat schematically, the semiclassical expansion is

$$
\begin{aligned}
U\left(x_{2} ; x_{1}\right)_{\rho}= & e^{i S_{0}[\rho]} \int_{0}^{0} D \tilde{x} e^{\frac{i}{2} \delta^{2} S_{0}[\rho] \tilde{w}^{2}} \\
& \times\left(1+\delta^{3} S_{0}[\rho] \tilde{w}^{3} / 6+\ldots\right),
\end{aligned}
$$

where $\tilde{w}$ stands for both $\tilde{x}$ or $\tilde{N} . S_{0}$ is the action evaluated with the extremal solutions.

Since our focus here is on finding a trajectory rather than fluctuations, consider the zero-loop approximation: $U\left(x_{2} ; x_{1}\right)_{\rho}=e^{i S_{0}[\rho]}$. For $W$ this gives

$$
W\left[\rho, \rho^{\prime}\right]=-i \ln \left(e^{i S_{0}[\rho]-i S_{0}\left[\rho^{\prime}\right]}\right)=S_{0}[\rho]-S_{0}\left[\rho^{\prime}\right] .
$$

Recalling that $S_{0}[\rho]=S\left[x_{0}\right]+\int \rho_{\mu} \dot{x}_{0}^{\mu} d \tau$, it follows that

$$
\left\langle\dot{x}^{\mu}\right\rangle=-\left.i \frac{\delta W}{\delta \rho_{\mu}}\right|_{\rho=0}=\dot{x}_{0}^{\mu} .
$$

In other words, the quantum-average trajectory (at zeroloop) is just the classical (extremal) solution.

It is instructive to consider the effective action at this same order, defined by the Legendre transformation

$$
\begin{aligned}
\Gamma\left[\left\langle\dot{x}^{\mu}\right\rangle\right] & \equiv W\left[\rho, \rho^{\prime}\right]-\int \rho_{\mu}\left\langle\dot{x}^{\mu}\right\rangle d \tau+\int \rho_{\mu}^{\prime}\left\langle\dot{x}^{\prime \mu}\right\rangle d \tau \\
& =S[\langle x\rangle]-S\left[\left\langle x^{\prime}\right\rangle\right] .
\end{aligned}
$$

The effective action is thus the classical action at zero-loop, and the equations of motion

$$
\frac{\delta \Gamma[\langle\dot{x}\rangle]}{\delta\left\langle\dot{x}^{\mu}\right\rangle}=\frac{\delta S\left[x_{0}\right]}{\delta \dot{x}_{0}^{\mu}}=0
$$

are the classical equations of motion. Including higherorder (loop) contributions there will generally be both quantum corrections to $\left\langle\dot{x}^{\mu}\right\rangle$ away from $x_{0}$, and higherorder correlation functions representing fluctuations.

\section{CONCLUSION}

For a relativistic particle moving in a background electromagnetic field the result Eq. 3 still holds but with $S_{\text {int }}=e \int d \tau \dot{x}^{\mu} A_{\mu}(x)$ added to the action. Otherwise, our above analysis carries through with straightforward substitutions. In [4], we show how to include the field's quantum dynamics in addition to an external classical background field. In this way we obtain QED as a theory of quantized worldlines interacting with the quantum electrodynamic field. Within this framework, we derive a modified Abraham-Lorentz-Dirac (ALD) equation after making semiclassical approximations to the path integrals. The ALD equation is modified at very short times due to timedependent mass and charge renormalization effects resulting from the nonequilibrium quantum dynamics of the interacting particle-field system. These nonequilibrium effects give causal and self-consistent equations of motion depending on the cutoff used in regularizing the theory, but runaway solutions reappear if the cutoff is made too large. In this respect the results are quite reminiscent of the classical radiation reaction for extended charge particles.

In this paper, we have seen how using worldline path integral quantization to define a generating function for worldline expectation values succeeds in producing equations of motion for a relativistic particle's quantum average trajectory from quantum theory. The approach systematically generalizes to incorporate quantum corrections (to the average) and quantum fluctuations (around the average). But as cautionary note, we must recognize that for highly non-classical particle states the equations of motion for the average may be very different than what one finds on making a measurement on the state. For instance, a wavepacket that is a superposition of two widely separate packets will give an average position in between the two packets even though actual individual measurements will essentially never find the particle in such a region.

\section{REFERENCES}

[1] P.R. Johnson and A.J. Dragt, The Propagation of Quantum Relativistic Wavepackets in Electromagnetic Fields, These proceedings, (2001).

[2] C. Schubert, Acta. Phys. Pol. B 27, p. 3965 (1996).

[3] M. Green, J. Schwarz, and E. Witten, Superstring Theory, Cambridge University Press (1987).

[4] P.R. Johnson and B.L. Hu, Stochastic Theory of Relativistic Particles Moving in a Quantum Field: I. Influence Functional and Langevin Equation, quant-ph/0013237, (2001); Stochastic Theory of Relativistic Particles Moving in a Quantum Field: II. Scalar Abraham-Lorentz-Dirac-Langevin Equation, Radiation Reaction, and Vacuum Fluctuations, quantph/0101001, (2001); Stochastic Theory of Relativistic Particle Motion Derived From Worldline QED, Preprint (2001); Worldline Influence Functional: Abraham-Lorentz-DiracLangevin Equation from QED, to appear in the proceedings of the second conference on Quantum Aspects of Beam Physics, edited by Pisin Chen, World Scientific, Singapore (2001), quant-ph/0012135. 